\title{
Relationship between metabolic syndrome and thyroid nodules in healthy Koreans
}

\author{
Juyoung Shin, Min-Hee Kim, Kun-Ho Yoon, Moo-Il Kang, Bong-Yun Cha, and Dong-Jun Lim
}

Division of Endocrinology and Metabolism, Department of Internal Medicine, College of Medicine, The Catholic University of Korea, Seoul, Korea

Received: April 28, 2014

Revised : January 23, 2015

Accepted: May 13, 2015

\section{Correspondence to}

Dong-Jun Lim, M.D.

Division of Endocrinology and Metabolism, Department of Internal Medicine, College of Medicine, The Catholic University of Korea, 222 Banpo-daero, Seocho-gu, Seoul 06591, Korea

Tel: +82-2-2258-6009

Fax: $+82-2-599-3589$

E-mail:1dj6026@catholic.ac.kr

A portion of these results were presented in abstract form at the 2013 Seoul International Congress of Endocrinology and Metabolism in Seoul, Korea.
Background/Aims: This study evaluated the relationship between thyroid nodules and metabolic syndrome (MS) and its components in apparently healthy Koreans.

Methods: We reviewed the records of 3,298 subjects with no noticeable symptoms who underwent thyroid ultrasound imaging as part of a routine check-up between July 2009 and June 2010; of these, 1,308 were excluded based upon predefined criteria. Among the remaining 1,990 patients, we examined the association between MS and its components and the incidence of thyroid nodules.

Results: Of the 1,990 subjects included in this study, 38.4\% $(n=764)$ had thyroid nodules and $12.7 \%(n=253)$ had MS. Female sex, older age, higher body mass index, larger waist circumference, higher glycated hemoglobin level, lower thyroid stimulating hormone level, and presence of MS were all closely related with the presence of thyroid nodules (all $p<0.05$ ). Furthermore, the relevant number of MS components showed a positive linear correlation with the occurrence of thyroid nodules $(p<0.001)$. Evidence of MS alone was not independently associated with thyroid nodules after adjusting for sex and age in a multivariate binary logistic regression analysis; however, glycated hemoglobin for females and waist circumference for males, as well as both age and thyroid stimulating hormone for all patients, were identified as independent predictors for the existence of thyroid nodules (all $p<0.05)$.

Conclusions: This study suggests a positive relationship between the components of MS and thyroid nodules in an ostensibly healthy Korean population. Our data support the idea that the recent increase in thyroid nodules is partly due to increases in both MS and obesity.

Keywords: Thyroid nodule; Metabolic syndrome; Obesity

\section{INTRODUCTION}

The incidence of thyroid nodules and subsequent thyroid cancer has increased steadily over the last 30 years. Some of this increase may be due to advances in diagnostic tools, including high-resolution ultrasonography and computed tomography, allowing for greater detection of thyroid nodules, which may not have been de- tected before [1]; however, important changes in the intrinsic characteristics of the general population are also assumed to be involved [2].

Metabolic syndrome (MS) is characterized by a cluster of metabolic risk factors, including central obesity, hypertension, hyperglycemia, and dyslipidemia [3]. As modern industrialization has brought a nutritional surplus and sedentary lifestyles to the general popula- 
tion, the prevalence of MS has been rising exponentially worldwide. Recent data have demonstrated that MS is a risk factor not only for cardiovascular disease and diabetes [4] but also for tumors, including breast, colorectal, pancreatic, and prostate cancers [5-8].

Several previous studies have demonstrated positive associations between MS and thyroid nodules, both benign and malignant, in the general population in Western countries [9,10]. However, it is not known whether a similar association exists among Asian populations. Here, we designed a cross-sectional study to evaluate the relationship between thyroid nodules and MS and its components in ostensibly healthy Koreans.

\section{METHODS}

\section{Study subjects}

We retrospectively reviewed the records of all participants older than 18 years who underwent thyroid ultrasound exams as part of a routine medical check-up at the Health Promotion Center of Seoul St. Mary's Hospital, a major tertiary hospital in Seoul, Korea, from July 2009 to June 2010. Among the 3,298 asymptomatic subjects identified in our initial screen, we excluded subjects who had missing data in questionnaires; had a different racial or ethnic background; had a self-reported history of diabetes, cardiovascular, or thyroid disease; took lipid-lowering agents such as statins or omega-3 fatty acids; or showed abnormalities on thyroid function tests. Ethical approval for the study protocol and data analysis was obtained from the Institutional Review Board of Seoul St. Mary's Hospital.

\section{Measurement of anthropometric and biochemical parameters}

Height and weight were measured without shoes and while wearing light indoor clothes by trained healthcare personnel. Body mass index was calculated as weight divided by height squared $\left(\mathrm{kg} / \mathrm{m}^{2}\right)$. Systolic and diastolic blood pressure readings were taken in a sitting position with an automatic device after a 10-minute rest. Waist circumference (WC) was measured at the level above the iliac crest, as defined by National Cholesterol Education Program's Adult Treatment Panel III (NCEP-ATPIII) guidelines [11].
Blood was drawn after an overnight fast. Fasting plasma glucose (FPG) levels were measured in serum with a modified glucose oxidase/peroxidase method (Sekisui Medical, Tokyo, Japan). Glycated hemoglobin (HbAic) levels were measured with a G7 glycohemoglobin analyzer (Tosoh Bioscience, Tokyo, Japan). Total cholesterol (TC), triglycerides (TGs), high density lipoprotein cholesterol (HDL-C), and low density lipoprotein cholesterol (LDL-C) were determined using standard enzymatic methods (Sekisui Medical). Thyroid stimulating hormone (TSH) and free thyroxine were measured with an electrochemiluminescence immunoassay (Elecsys 1010, Roche, Indianapolis, IN, USA).

MS was defined as the condition in which three or more of the following metabolic components were satisfied, according to NCEP-ATPIII guidelines: (1) WC $\geq$ $90 \mathrm{~cm}$ in males, $\geq 80 \mathrm{~cm}$ in females; (2) TG $\geq 150 \mathrm{mg} / \mathrm{dL}$; (3) $\mathrm{HDL}-\mathrm{C}<40 \mathrm{mg} / \mathrm{dL}$ in males, $<50 \mathrm{mg} / \mathrm{dL}$ in females; (4) blood pressure $\geq 130 / 85 \mathrm{mmHg}$ or taking antihypertensive medication; and (5) FPG $\geq 100 \mathrm{mg} / \mathrm{dL}$.

\section{Thyroid evaluation}

Thyroid ultrasonography was performed by radiologists using an HDI 5000 (Phillips Ultrasound, Bothell, WA, USA) with a 7-15 MHz transducer, according to the standard protocol of the health promotion center. The diagnosis of thyroid nodules was obtained from a review of radiological reports. A thyroid nodule was defined as discrete lesions distinct from the surrounding thyroid parenchyma, and which had a solid portion regardless of the presence of a cystic portion. The maximal diameter of a thyroid nodule was measured in three planes: longitudinal, transverse, and anteroposterior.

\section{Statistical analysis}

Categorical data are expressed as numbers and percentages of subjects. Continuous variables are expressed as the mean \pm standard deviation. To evaluate differences in metabolic parameters between those with and without thyroid nodules, categorical variables were analyzed based on the chi-square test and continuous variables were compared with the unpaired $t$ test. To find any association between metabolic components and the prevalence of thyroid nodules, we analyzed the data using a binary logistic regression analysis. A multivariate binary logistic regression analysis was used to determine inde- 
pendent factors for predicting the occurrence of thyroid nodules. A $p<0.05$ was considered statistically significant. All analyses were performed using SPSS version 15.0 (SPSS Inc., Chicago, IL, USA).

\section{RESULTS}

\section{Clinical characteristics of the study subjects}

A total of 3,298 subjects were identified who underwent thyroid ultrasound exams as part of a general health check-up. Subjects were excluded from analysis based upon: (1) missing data in patient questionnaires $(\mathrm{n}=156)$; (2) unknown race or ethnicity $(\mathrm{n}=63)$; (3) known thyroid disease ( $n=180$ ); (4) use of lipid-lowering agents, including statins or omega-3 fatty acids $(n=279) ;(5)$ history of diabetes $(n=175)$ or cardiovascular disease $(n=123)$; or $(6)$ thyroid dysfunction $(\mathrm{n}=332$ ) (Fig. 1). Based upon these criteria, we were able to identify 1,990 subjects for inclusion in the final study population.

\section{Metabolic variables and thyroid nodules}

The baseline characteristics of the study subjects are summarized in Tables 1 and 2. Among the 1,990 subjects in the final analysis, 764 (38.4\%) had thyroid nodules and 253 (12.7\%) had MS. The study population consisted of 1,299 females (65.3\%) and had a mean age of $49.8 \pm 10.0$ years.

Subjects with thyroid nodules showed a higher female to male ratio (70.8\% vs. $61.8 \%, p<0.001)$ and older age $(52.4 \pm 9.5$ years vs. $48.2 \pm 10.0$ years, $p<0.001)$ relative

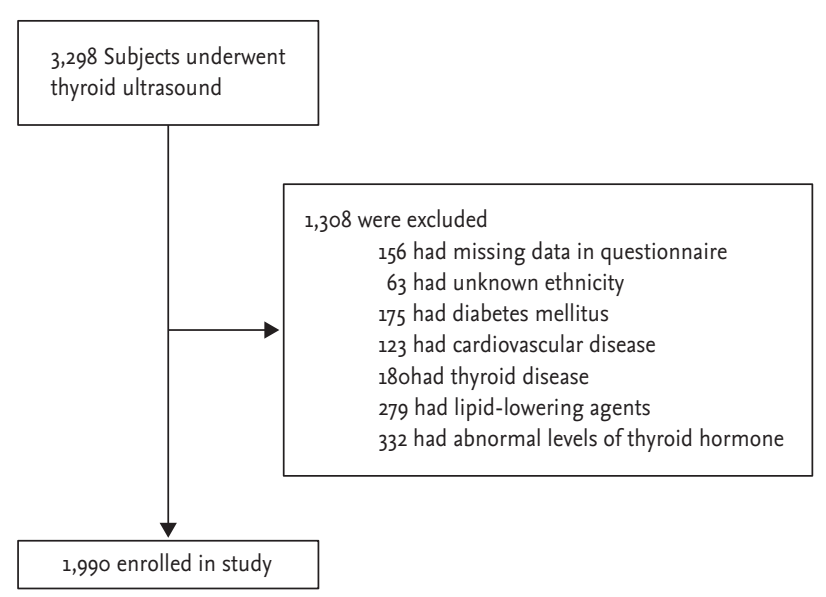

Figure 1. Selection of study participants.

Table 1. Metabolic variables and presence of thyroid nodules in subjects with and without thyroid nodules

\begin{tabular}{|c|c|c|c|c|}
\hline Variable & $\begin{array}{c}\text { Total } \\
(\mathrm{n}=1,990)\end{array}$ & $\begin{array}{l}\text { With thyroid nodule } \\
\qquad(\mathrm{n}=764)\end{array}$ & $\begin{array}{l}\text { Without thyroid nodule } \\
\qquad(\mathrm{n}=1,226)\end{array}$ & $p$ value ${ }^{a}$ \\
\hline Male sex & $691(34.7)$ & $223(29.2)$ & $468(38.2)$ & $<0.001$ \\
\hline Age, yr & $49.8 \pm 10.0$ & $52.4 \pm 9.5$ & $48.2 \pm 10.0$ & $<0.001$ \\
\hline Systolic blood pressure, mmHg & $118.4 \pm 14 \cdot 3$ & $119.2 \pm 14.3$ & $118.0 \pm 14 \cdot 3$ & 0.073 \\
\hline Diastolic blood pressure, $\mathrm{mmHg}$ & $69.2 \pm 10.1$ & $69.5 \pm 10.2$ & $69.0 \pm 10.1$ & 0.279 \\
\hline Body mass index, $\mathrm{kg} / \mathrm{m}^{2}$ & $22.9 \pm 3.1$ & $23.2 \pm 3.0$ & $22.7 \pm 3.1$ & 0.001 \\
\hline Waist circumference, $\mathrm{cm}$ & $82.3 \pm 8.1$ & $83.2 \pm 8.1$ & $81.8 \pm 8.1$ & $<0.001$ \\
\hline Fasting plasma glucose, mg/dL & $87.0 \pm 15.2$ & $87.7 \pm 16.3$ & $86.5 \pm 14.5$ & 0.083 \\
\hline Glycated hemoglobin, \% & $5.5 \pm 0.5$ & $5.6 \pm 0.6$ & $5.5 \pm 0.5$ & $<0.001$ \\
\hline Total cholesterol, mg/dL & $199.2 \pm 34.1$ & $199.6 \pm 33.9$ & $198.9 \pm 34.3$ & 0.644 \\
\hline Triglyceride, mg/dL & $93.7 \pm 66.2$ & $94.6 \pm 59.0$ & $93.1 \pm 70.3$ & 0.619 \\
\hline HDL-C, mg/dL & $55.2 \pm 13.1$ & $54.8 \pm 12.6$ & $55 \cdot 5 \pm 13 \cdot 4$ & 0.253 \\
\hline LDL-C, mg/dL & $120.0 \pm 31.1$ & $120.3 \pm 31.1$ & $119.8 \pm 31.1$ & 0.757 \\
\hline TSH, mIU/L & $2.1 \pm 1.1$ & $2.0 \pm 1.0$ & $2.2 \pm 1.1$ & $<0.001$ \\
\hline Free thyroxine, ng/dL & $1.3 \pm 0.2$ & $1.3 \pm 0.2$ & $1.3 \pm 0.2$ & 0.883 \\
\hline Metabolic syndrome & $253(12.7)$ & $115(15.1)$ & $138(11.3)$ & 0.015 \\
\hline
\end{tabular}

Values are presented as number (\%) or mean \pm SD.

HDL-C, high density lipoprotein cholesterol; LDL-C, low density lipoprotein cholesterol; TSH, thyroid stimulating hormone. ${ }^{a}$ With thyroid nodule vs. without thyroid nodule. 
Table 2. Metabolic variables and presence of thyroid nodules in subjects with and without metabolic syndrome

\begin{tabular}{|c|c|c|c|c|}
\hline Variable & $\begin{array}{c}\text { Total } \\
(\mathrm{n}=1,990)\end{array}$ & $\begin{array}{l}\text { With metabolic syndrome } \\
\qquad(\mathrm{n}=253)\end{array}$ & $\begin{array}{l}\text { Without metabolic syndrome } \\
\qquad(\mathrm{n}=1,737)\end{array}$ & $p$ value $^{\mathrm{a}}$ \\
\hline Male sex & $691(34.7)$ & $109(43.1)$ & $582(33.5)$ & 0.004 \\
\hline Age, yr & $49.8 \pm 10.0$ & $54.9 \pm 9.0$ & $49.1 \pm 9.9$ & 0.058 \\
\hline Systolic blood pressure, $\mathrm{mmHg}$ & $118.4 \pm 14.3$ & $129.8 \pm 15.5$ & $116.8 \pm 13.3$ & $<0.001$ \\
\hline Diastolic blood pressure, $\mathrm{mmHg}$ & $69.2 \pm 10.1$ & $76.3 \pm 10.2$ & $68.1 \pm 9.7$ & $<0.001$ \\
\hline Body mass index, kg/m² & $22.9 \pm 3.1$ & $25.8 \pm 2.8$ & $22.5 \pm 2.9$ & $<0.001$ \\
\hline Waist circumference, $\mathrm{cm}$ & $82.3 \pm 8.1$ & $90.3 \pm 6.9$ & $81.2 \pm 7.6$ & $<0.001$ \\
\hline Fasting plasma glucose, mg/dL & $87.0 \pm 15.2$ & $98.9 \pm 26.2$ & $85.2 \pm 11.8$ & $<0.001$ \\
\hline Glycated hemoglobin, \% & $5.5 \pm 0.5$ & $6.0 \pm 0.9$ & $5 \cdot 5 \pm 0.4$ & $<0.001$ \\
\hline Total cholesterol, mg/dL & $199.2 \pm 34.1$ & $208.3 \pm 39.5$ & $197.8 \pm 33.1$ & $<0.001$ \\
\hline Triglyceride, mg/dL & $93.7 \pm 66.2$ & $173.8 \pm 102.4$ & $82.1 \pm 49 \cdot 3$ & $<0.001$ \\
\hline $\mathrm{HDL}-\mathrm{C}, \mathrm{mg} / \mathrm{dL}$ & $55.2 \pm 13.1$ & $42.8 \pm 8.8$ & $57.0 \pm 12.6$ & $<0.001$ \\
\hline LDL-C, mg/dL & $120.0 \pm 31.1$ & $128.3 \pm 36.0$ & $118.8 \pm 30.1$ & $<0.001$ \\
\hline TSH, mIU/L & $2.1 \pm 1.1$ & $2.2 \pm 1.1$ & $2.1 \pm 1.1$ & 0.497 \\
\hline Free thyroxine, ng/dL & $1.3 \pm 0.2$ & $1.3 \pm 0.2$ & $1.3 \pm 0.2$ & 0.592 \\
\hline Thyroid nodule & $764(38.4)$ & $115(45 \cdot 5)$ & $649(37.4)$ & 0.015 \\
\hline Thyroid nodule size $\geq 1 \mathrm{~cm}$ & $186 / 764(24 \cdot 3)$ & $38 / 115(33.0)$ & $148 / 649(22.8)$ & 0.002 \\
\hline Thyroid nodule multiplicity & $297 / 764(38.9)$ & $49 / 115(42.6)$ & $248 / 649(38.2)$ & 0.346 \\
\hline
\end{tabular}

Values are presented as number (\%) or mean \pm SD.

HDL-C, high density lipoprotein cholesterol; LDL-C, low density lipoprotein cholesterol; TSH, thyroid stimulating hormone. ${ }^{a}$ With metabolic syndrome vs. without metabolic syndrome.

Table 3. Multivariate binary logistic regression analysis of independent predictors for the presence of thyroid nodules $(\mathrm{n}=1,635)$

\begin{tabular}{|c|c|c|}
\hline Variable & Odds ratio & $p$ value \\
\hline \multicolumn{3}{|l|}{ Female sex $(n=1,140)$} \\
\hline Age, yr & 1.059 & $<0.001$ \\
\hline Systolic blood pressure, mmHg & 0.989 & 0.151 \\
\hline Diastolic blood pressure, $\mathrm{mmHg}$ & 1.005 & 0.635 \\
\hline Waist circumference, $\mathrm{cm}$ & 1.011 & 0.283 \\
\hline Glycated hemoglobin, \% & 1.512 & 0.011 \\
\hline Triglyceride, mg/dL & 0.999 & 0.245 \\
\hline HDL-C, mg/dL & 0.995 & 0.360 \\
\hline TSH, mIU/L & 0.828 & 0.001 \\
\hline \multicolumn{3}{|l|}{ Male sex $(n=595)$} \\
\hline Age, yr & 1.042 & $<0.001$ \\
\hline Systolic blood pressure, $\mathrm{mmHg}$ & 0.989 & 0.352 \\
\hline Diastolic blood pressure, $\mathrm{mmHg}$ & 1.005 & 0.741 \\
\hline Waist circumference, $\mathrm{cm}$ & 1.042 & 0.005 \\
\hline Glycated hemoglobin, \% & 0.951 & 0.734 \\
\hline Triglyceride, mg/dL & 1.000 & 0.780 \\
\hline HDL-C, mg/dL & 0.999 & 0.882 \\
\hline TSH, mIU/L & 0.714 & $<0.001$ \\
\hline
\end{tabular}

HDL-C, high density lipoprotein cholesterol; TSH, thyroid stimulating hormone. 


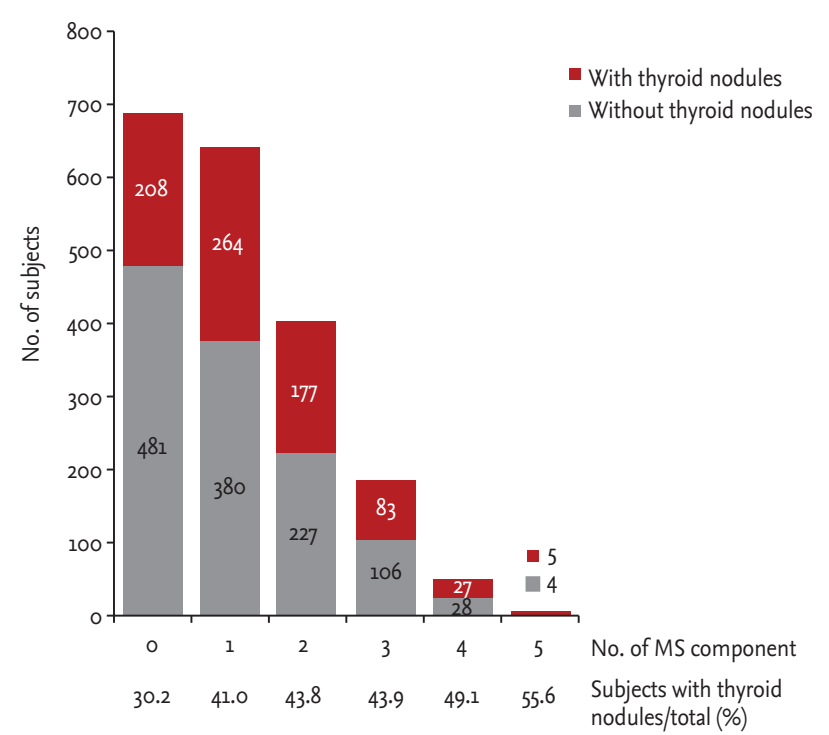

Figure 2. Association between the number of relevant components of metabolic syndrome (MS) and prevalence of thyroid nodules.

to those without thyroid nodules. The prevalence of MS was also higher in subjects with thyroid nodules than in those without (115/764 [15.1\%] vs. 138/1,226 [11.3\%], $p=$ o.015). Body mass index, WC, and HbAic were significantly higher in subjects with thyroid nodules, while blood pressure, FPG, and lipid profiles, including TC, TG, HDL-C, and LDL-C, showed no statistically significant difference between groups. Subjects with MS had a higher prevalence of not only thyroid nodules but also nodules larger than $1 \mathrm{~cm}$ (Table 2). However, the prevalence of multiple thyroid nodules did not differ between subjects with or without MS.

Subjects with MS showed a lower female to male ratio (56.9\% vs. $66.5 \%, p=0.004)$ and a higher prevalence of thyroid nodules ( $45.5 \%$ vs. $37.4 \%, p=0.015$ ) than those without MS. The mean age of the subjects with MS was higher, though this difference was not statistically significant $(54.9 \pm 9.0$ years vs. $49.1 \pm 9.9$ years, $p=0.058)$.

As the number of MS components satisfied increased, the proportion of patients with thyroid nodules rose, indicating a strong correlation between MS and thyroid nodules $(\beta=1.235, p<0.001$ ) (Fig. 2). However, individual components of MS did not show independent relationships with thyroid nodules after adjusting for sex and age. Moreover, age stratification showed that the prevalence of both thyroid nodules and MS was closely associated with age (Supplementary Fig. 1). To address these apparent discrepancies, we ran a multivariate binary logistic regression analysis to identify clinical variables that could independently predict the existence of thyroid nodules (Table 3). HbAic levels were a significant predictor of thyroid nodules in females $(p=0.011)$, while WC was a significant predictor only in males $(p=0.005)$; higher age and lower TSH were significant factors for predicting thyroid nodules in both sexes $(p<0.001)$.

\section{DISCUSSION}

In this study, we explored the relationship between the presence of thyroid nodules and the components of MS in ostensibly healthy Koreans with no evidence of iodine insufficiency [12]. Our results suggest an association between thyroid nodules and MS components even in healthy populations, which may have important implications for the diagnosis and treatment of thyroid-related diseases.

MS is closely associated with an increase in overall mortality, due not only to increases in cardiovascular complications [4], but also malignant diseases [5-8]. Insulin resistance, a characteristic manifestation of MS, increases glucose and insulin levels, which stimulate cell proliferation. Large population studies revealed that patients with glucose metabolism disorders have a higher chance of both malignancy and mortality [5,1316]. The tumorigenic effects of MS appear to be mediated through changes in cytokine levels, resulting in decreased expression of tumor suppression genes, and increases in oncogene expression due to inflammation and reactive oxygen species generation; other factors, such as the stimulation of angiogenesis and invasion of malignant cells into normal tissues, may also play a role [5]. While many of the mechanisms underlying these effects remain poorly understood, these activities are well supported by a variety of observational and laboratory studies.

Recent studies suggest that components of MS are possible risk factors for thyroid nodules, beyond many of the well-established ones, including sex, goiter, family history of thyroid cancer, and radiation exposure. MS is thought to trigger thyroid nodules via the stimulation of thyroid proliferation and angiogenesis brought on by hyperinsulinemia, hyperglycemia, and dyslipidemia [17- 
19]. Our data demonstrate that people with MS had a higher prevalence of thyroid nodules. The subjects with MS also had a higher prevalence of thyroid nodules larger than $1 \mathrm{~cm}$, consistent with a previous study showing a positive association between insulin resistance and thyroid nodule size [19]. Furthermore, we showed that the number of accompanying MS factors had a significant positive correlation with the prevalence of thyroid nodules; the more components of MS were observed, the higher the chance of thyroid nodules. The prevalence of thyroid nodules may be affected by sex [20,21]; as older age was also positively correlated with not only the presence of thyroid nodules, but also MS [22], we analyzed the role of sex independently using multivariate logistic regression. After adjusting for age, higher $\mathrm{HbAlc}$ in females and larger WC in males were found to be significant factors for the presence of thyroid nodules.

Many previous studies have shown that hyperglycemia has a strong correlation with thyroid cancer in both sexes $[23,24]$. Our study demonstrates that hyperglycemia, as indicated by $\mathrm{HbAcc}$ levels, showed a significant relationship with the presence of thyroid nodules in women. An interesting finding is that $\mathrm{HbArc}$ levels showed a significant association with thyroid nodules both before and after adjustment for related factors, whereas FPG levels did not. The exclusion of all known diabetic patients to target a metabolically normal population might make these two hyperglycemia-related parameters contradictory. Consistent with our study, a previous univariate analysis of an Icelandic cohort showed the relative risk for women with high 90-minute postprandial glucose levels for thyroid cancer was 1.12 [14], indicating that postprandial glucose levels may be associated with thyroid nodules and, potentially, thyroid cancer. Although the effects of postprandial hyperglycemia on tumor formation have been not well evaluated, hyperglycemia, oxidative stress, endothelial dysfunction, and the formation of advanced glycation end products may all potentially be involved in the pathophysiology of tumor formation $[5,25,26]$. As HbAic levels are routinely used to screen for the existence of diabetes [27-30], our center was able to measure these levels across all patients; however, we were unable to evaluate insulin resistance outcomes, such as homeostasis model assessment of insulin resistance, as neither insulin nor c-peptide were routinely measured.
Anthropometric measurements showed inconsistent associations with the prevalence of thyroid cancer. Most studies that have evaluated the relationship between body mass index and thyroid cancer have reported a strong association between them [14,31,32]; however, the significance varied with sex. Kitahara et al. [33] demonstrated a slightly stronger association of WC and weight change with thyroid cancer risk in men versus women, which may be due to a difference in hormone profiles, such as leptin and estrogen, and the metabolic consequences of excess body fat in men and women.

TSH levels were inversely related with thyroid nodules, even though both TSH levels were within normal ranges. One possible explanation is that multiple thyroid nodules could maintain TSH levels at a lower normal range by autonomous hyperfunctioning. TSH has been shown to be suppressed in patients with a multinodular goiter [34], while TSH levels < $2.5 \mathrm{mIU} / \mathrm{L}$ may be related to an increased risk of MS [35]. These associations were not replicated in our study, with MS patients showing no increases in TSH levels (Table 2). This difference may be an artifact of the study design, as our study population consisted entirely of patients who underwent thyroid ultrasound imaging.

Our study has several unique clinical features. The most important aspect of this study is its use of clinically healthy subjects. Because we initially excluded all subjects with a history of diabetes or cardiovascular disease and those taking lipid-lowering agents, the prevalence of MS (12.7\%) in our study population was much lower than that in the general Korean population, with MS prevalence rates estimated at 31.3\% [36]. Although the strict inclusion criteria may have underestimated the effects of abnormal glucose and lipid metabolism on the thyroid, MS components showed significant connections with thyroid nodules. Second, this analysis was based on reliable parameters measured by specialists using standardized methods at a single center. The large number of subjects enrolled is also an important strength of this study.

Despite its many strengths, this study is not without limitations. First, it was a retrospective, cross-sectional study. Therefore, the study population might be biased, as the study population consisted of people who determined by themselves whether they should undergo thyroid ultrasonography. This aspect of the study de- 
sign may explain why the prevalence of thyroid nodules was similar between sexes, unlike a previous study [21]. A longitudinal cohort study with a more representative population will be needed to address these potential issues. Second, we could not evaluate the association between insulin resistance and thyroid nodules because insulin and C-peptide levels were not available. A lack of cytological or histological results for each nodule is an important limitation. Future studies focusing on more biopsy-proven reports and with a longer follow-up duration will be necessary to provide a more complete and accurate evaluation of the relationship.

This study suggests an association between the components of MS and thyroid nodules in a healthy Korean population. Our data show that female sex, older age, lower TSH, and higher number of accompanying MS components were all positively associated with the presence of thyroid nodules. Future studies evaluating the effects of active interventions, including lifestyle modification and medication adjustments, on components of MS with regard to the incidence of thyroid nodules and, ultimately, the prevention of thyroid cancer, are therefore warranted.

\section{KEY MESSAGE}

1. There is a positive relationship between the components of metabolic syndrome (MS) and thyroid nodules in an ostensibly healthy Korean population.

2. A recent increase in thyroid nodules is partly due to increases in both MS and obesity.

\section{Conflict of interest}

No potential conflict of interest relevant to this article was reported.

\section{REFERENCES}

1. Davies L, Welch HG. Increasing incidence of thyroid cancer in the United States, 1973-2002. JAMA 2006;295:21642167.

2. Enewold L, Zhu K, Ron E, et al. Rising thyroid cancer incidence in the United States by demographic and tumor characteristics, 1980-2005. Cancer Epidemiol Biomarkers Prev 2009;18:784-791.

3. Eckel RH, Grundy SM, Zimmet PZ. The metabolic syndrome. Lancet 2005;365:1415-1428.

4. Lorenzo C, Williams K, Hunt KJ, Haffner SM. The National Cholesterol Education Program-Adult Treatment Panel III, International Diabetes Federation, and World Health Organization definitions of the metabolic syndrome as predictors of incident cardiovascular disease and diabetes. Diabetes Care 2007;30:8-13.

5. Pothiwala P, Jain SK, Yaturu S. Metabolic syndrome and cancer. Metab Syndr Relat Disord 2009;7:279-288.

6. Esposito K, Chiodini P, Capuano A, et al. Metabolic syndrome and postmenopausal breast cancer: systematic review and meta-analysis. Menopause 2013;20:1301-1309.

7. Russo A, Autelitano M, Bisanti L. Metabolic syndrome and cancer risk. Eur J Cancer 2008;44:293-297.

8. Lund Haheim L, Wisloff TF, Holme I, Nafstad P. Metabolic syndrome predicts prostate cancer in a cohort of middle-aged Norwegian men followed for 27 years. Am J Epidemiol 2006;164:769-774.

9. Ayturk S, Gursoy A, Kut A, Anil C, Nar A, Tutuncu NB. Metabolic syndrome and its components are associated with increased thyroid volume and nodule prevalence in a mild-to-moderate iodine-deficient area. Eur J Endocrinol 2009;161:599-605.

10. Rezzonico JN, Rezzonico M, Pusiol E, Pitoia F, Niepomniszcze $H$. Increased prevalence of insulin resistance in patients with differentiated thyroid carcinoma. Metab Syndr Relat Disord 2009;7:375-380.

11. Expert Panel on Detection, Evaluation, and Treatment of High Blood Cholesterol in Adults. Executive summary of the third report of the National Cholesterol Education Program (NCEP) expert panel on detection, evaluation, and treatment of high blood cholesterol in adults (Adult Treatment Panel III). JAMA 2001;285:2486-2497.

12. Kim JY, Kim KR. Dietary iodine intake and urinary iodine excretion in patients with thyroid diseases. Yonsei Med J 2000;41:22-28.

13. Saydah SH, Loria CM, Eberhardt MS, Brancati FL. Abnormal glucose tolerance and the risk of cancer death in the United States. Am J Epidemiol 2003;157:1092-1100.

14. Tulinius H, Sigfusson N, Sigvaldason H, Bjarnadottir K, Tryggvadottir L. Risk factors for malignant diseases: a cohort study on a population of 22,946 Icelanders. Cancer Epidemiol Biomarkers Prev 1997;6:863-873. 
15. Calle EE, Kaaks R. Overweight, obesity and cancer: epidemiological evidence and proposed mechanisms. Nat Rev Cancer 2004;4:579-591.

16. Ibrahim YH, Yee D. Insulin-like growth factor-I and cancer risk. Growth Horm IGF Res 2004;14:261-269.

17. Iribarren C, Haselkorn T, Tekawa IS, Friedman GD. Cohort study of thyroid cancer in a San Francisco Bay area population. Int J Cancer 2001;93:745-750.

18. Yin J, Wang C, Shao Q, et al. Relationship between the prevalence of thyroid nodules and metabolic syndrome in the iodine-adequate area of Hangzhou, China: a cross-sectional and cohort study. Int J Endocrinol 2014;2014:675796.

19. Wang K, Yang Y, Wu Y, et al. The association between insulin resistance and vascularization of thyroid nodules. J Clin Endocrinol Metab 2015;100:184-192.

20. Mazzaferri EL. Management of a solitary thyroid nodule. N Engl J Med 1993;328:553-559.

21. Kim JY, Jung EJ, Park ST, et al. Body size and thyroid nodules in healthy Korean population. J Korean Surg Soc 2012;82:13-17.

22. Hildrum B, Mykletun A, Hole T, Midthjell K, Dahl AA. Age-specific prevalence of the metabolic syndrome defined by the International Diabetes Federation and the National Cholesterol Education Program: the Norwegian HUNT 2 study. BMC Public Health 2007;7:220.

23. Zhan YS, Feng L, Tang SH, et al. Glucose metabolism disorders in cancer patients in a Chinese population. Med Oncol 2010;27:177-184.

24. Zivaljevic V, Vlajinac H, Jankovic R, Marinkovic J, Diklic A, Paunovic I. Case-control study of anaplastic thyroid cancer. Tumori 2004;90:9-12.

25. Gerich JE. Clinical significance, pathogenesis, and management of postprandial hyperglycemia. Arch Intern Med 2003;163:1306-1316.

26. Heine RJ, Balkau B, Ceriello A, Del Prato S, Horton ES, Taskinen MR. What does postprandial hyperglycaemia mean? Diabet Med 2004;21:208-213.

27. Ko SH, Kim SR, Kim DJ, et al. 2011 Clinical practice guidelines for type 2 diabetes in Korea. Diabetes Metab J 2011;35:431-436.

28. International Expert Committee. International Expert Committee report on the role of the $\mathrm{AlC}$ assay in the diagnosis of diabetes. Diabetes Care 2009;32:1327-1334.

29. Saudek CD, Herman WH, Sacks DB, Bergenstal RM, Edelman D, Davidson MB. A new look at screening and diagnosing diabetes mellitus. J Clin Endocrinol Metab 2008;93:2447-2453.

30. Bennett CM, Guo M, Dharmage SC. $\mathrm{HbA}(1 \mathrm{c})$ as a screening tool for detection of type 2 diabetes: a systematic review. Diabet Med 2007;24:333-343.

31. Dal Maso L, La Vecchia C, Franceschi S, et al. A pooled analysis of thyroid cancer studies. V. Anthropometric factors. Cancer Causes Control 2000;11:137-144.

32. Engeland A, Tretli S, Akslen LA, Bjorge T. Body size and thyroid cancer in two million Norwegian men and women. Br J Cancer 2006;95:366-370.

33. Kitahara CM, Platz EA, Park Y, Hollenbeck AR, Schatzkin A, Berrington de Gonzalez A. Body fat distribution, weight change during adulthood, and thyroid cancer risk in the NIH-AARP Diet and Health Study. Int J Cancer 2012;130:1411-1419.

34. Elte JW, Bussemaker JK, Haak A. The natural history of euthyroid multinodular goitre. Postgrad Med J 1990;66:186-190.

35. Oh JY, Sung YA, Lee HJ. Elevated thyroid stimulating hormone levels are associated with metabolic syndrome in euthyroid young women. Korean J Intern Med 2013;28:180-186.

36. Lim S, Shin $\mathrm{H}$, Song JH, et al. Increasing prevalence of metabolic syndrome in Korea: the Korean National Health and Nutrition Examination Survey for 1998-2007. Diabetes Care 2011;34:1323-1328. 


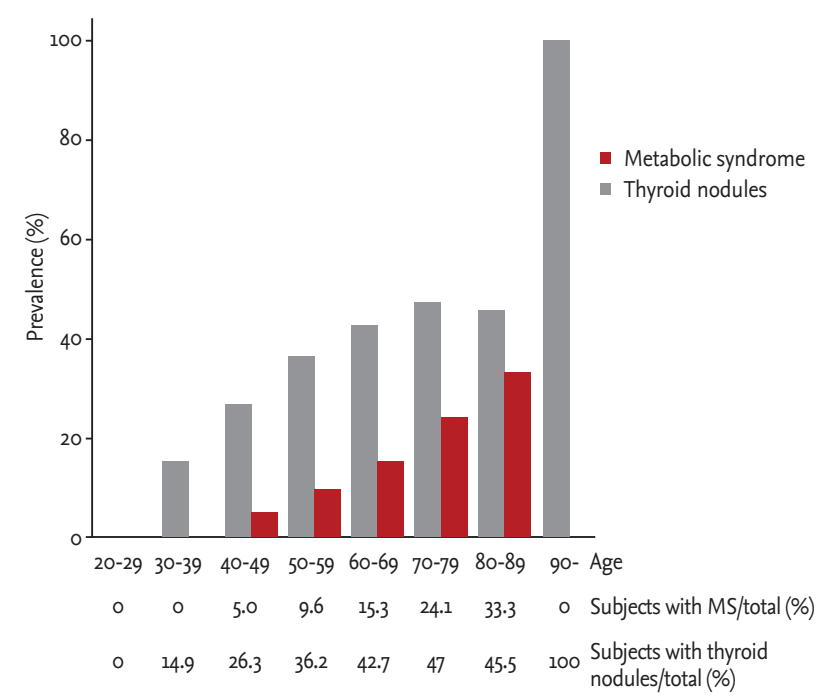

Supplementary Figure 1. Association between age and the prevalence of metabolic syndrome (MS) and thyroid nodules. 